\title{
Breakfast consumption and weight-loss maintenance: results from the MedWeight study
}

\author{
Dora Brikou, Dimitra Zannidi, Eleni Karfopoulou, Costas A. Anastasiou and Mary Yannakoulia* \\ Department of Nutrition E Dietetics, Harokopio University, 17671 Athens, Greece \\ (Submitted 15 September 2015 - Final revision received 6 March 2016 - Accepted 21 March 2016)
}

\begin{abstract}
Daily breakfast consumption is a common eating behaviour among people who have maintained their weight loss after weight-loss management. However, there is not a precise definition for breakfast in the literature. The purpose of this study was to investigate potential associations between breakfast consumption (based on several definitions) and weight-loss maintenance, as well as to explore differences in breakfast quality between individuals who managed to maintain part of the weight loss and in those who regained weight loss. The study sample consisted of 354 participants of the MedWeight study (age: 32 (sD 10) years, $61 \%$ women) who had lost $\geq 10 \%$ of their initial body weight and either maintained the loss for $\geq 1$ year (maintainers, $n$ 257) or regained weight loss (regainers, $n$ 97). Participants completed online questionnaires and reported their dietary intake through two telephone 24-h recalls. Breakfast consumption was evaluated using twelve different definitions. The analysis indicated that breakfast consumption was associated with weight-loss maintenance only in men, when using self-reported breakfast consumption or the following breakfast definitions: (1) the first eating episode consumed at home and (2) the first eating episode consumed at home excluding caffeinated drinks. This association remained statistically significant even after adjustment for potential confounding factors. Thus, breakfast, the first eating episode of the day, when consumed at home, may be protective against weight regaining.
\end{abstract}

Key words: Breakfast consumption: Meal patterns: Weight-loss maintenance: Obesity

Breakfast has been widely advocated as an important part of a healthy diet and as an effective strategy for achieving and maintaining a healthy weight ${ }^{(1,2)}$. However, supporting evidence is not as strong as one may believe ${ }^{(3,4)}$. Epidemiological data have associated breakfast skipping with increased risk of obesity in both children ${ }^{(5)}$ and adults $^{(6)}$, and have associated breakfast consumption with reduced risk of weight gain ${ }^{(7)}$. In addition, the percentage of total energy intake consumed at breakfast is negatively associated with weight gain ${ }^{(8)}$, and daily breakfast consumption is a common behaviour among weightloss maintainers ${ }^{(9)}$. Despite these findings, results from clinical trials tend to be conflicting. A recommendation to eat or skip breakfast failed to achieve a detectable effect on weight loss in free-living adults who were attempting to lose weight ${ }^{(10)}$. However, it has been shown that high energy intake at breakfast combined with low energy intake at dinner can lead to greater weight loss as compared with the opposite pattern ${ }^{(11)}$. To add to the conflicting data, another clinical trial showed that a high-energy breakfast had no better effect on weight loss than a high-energy dinner did, but those who consumed a high-energy breakfast had better weight-loss maintenance outcomes compared with those consuming a high-energy dinner ${ }^{(12)}$.

These contradictory results may be, at least partly, explained by the variety of breakfast definitions used in the related literature. Nevertheless, there is limited consensus as to what constitutes the term breakfast meal ${ }^{(13)}$. Breakfast meal has been defined by weekly frequency, time of day, timing in relation to waking and daily activities, types of food or beverages consumed or amount of energy provided ${ }^{(14)}$. The lack of a standard definition means that measurement of breakfast consumption in research is subject to either participants' interpretations of what constitutes breakfast or varied definitions provided by researchers ${ }^{(14)}$.

Thus, we reviewed the relevant literature for the definitions of breakfast, and applied those used more commonly on people who lost weight and maintained (maintainers) or regained (regainers) the loss. Our purpose was to examine whether breakfast consumption, and specifically which type of breakfast, is associated with weight-loss maintenance. In addition, we aimed to study the quality of the breakfast meal both in maintainers and regainers.

\section{Methods}

\section{Study design and sample}

The current analysis included 354 participants from the MedWeight, a registry of people who lost weight and maintained it (maintainers) and people who lost weight but regained the loss (regainers). Full details of the study design have been described elsewhere ${ }^{(15)}$. In brief, inclusion criteria were age between 18 and 65 years, initial BMI of $25 \mathrm{~kg} / \mathrm{m}^{2}$ or more and 
an intentional loss at least $10 \%$ of starting weight. Volunteers maintaining the minimum $10 \%$ loss for at least 1 year at study entry were classified as maintainers. Those currently at a weight $\geq 95 \%$ of their maximum body weight were classified as regainers. Individuals with a current body weight between 90 and $95 \%$ of their maximum weight were excluded in order to avoid overlapping of groups. Current pregnancy was also an exclusion criterion. Eligible participants completed the study questionnaires, which was available to them through the MedWeight website (http://medweight.hua.gr). The study protocol was approved by the Harokopio University Ethics Committee.

From the 354 participants, 257 were maintainers (73\%) and ninety-seven were regainers (27\%). Descriptive characteristics of the participants are presented in Table 1. Maintainers were younger compared with regainers and achieved greater initial weight loss.

\section{Assessment of dietary intake}

Dietary intake was evaluated using the 24-h recall method ${ }^{(16)}$. Participants were asked to report in detail all foods and beverages consumed on the day before (i.e. between waking up in the morning and going to bed at night). Time, location, parallel activities and companions were also recorded for each eating occasion. Two telephone 24-h dietary recalls were performed in each volunteer ${ }^{(17)}, 10 \mathrm{~d}$ apart from each other, with weekdays and weekends proportionately represented among participants. Furthermore, participants did not know when these recalls would be occurring, so they could not change their diet in anticipation of the recall.

Recall data were analysed for energy, macro- and micronutrient intake using the dietary analysis software Nutritionist $\operatorname{Pro}^{\text {TM }}$ (2007, Axxya Systems). In addition, dietary intake was grouped into twenty-seven food groups, featuring the core foods of the Greek diet, as well as food items or groups associated with obesity ${ }^{(18,19)}$. Portion sizes used were according to the Mediterranean diet pyramid ${ }^{(20)}$.

Diet quality was assessed using the MedDietScore, a simple dietary score based on the characteristics of the Mediterranean diet. The score ranges from 0 to 55 , and higher values indicate greater adherence to the Mediterranean $\operatorname{diet}^{(21)}$.

\section{Breakfast assessment and definitions}

First, we reviewed current literature in order to locate the various definitions of breakfast used previously. A PubMed search was conducted for breakfast-related articles published until December 2013, using "breakfast" as a search term included in the title of the article. In total, 1023 articles were screened and fifty unique definitions of breakfast were identified. These definitions refer to one of the following categories: time of day, timing in relation to waking up and/or daily activities, time combined with energy provided, place of consumption, types of food or beverages consumed, combinations of these categories or self-reported breakfast. As a second step, we applied the most commonly used definitions of breakfast meal from each of the categories, and we also performed additional combinations - that is twelve breakfast definitions in total.

*

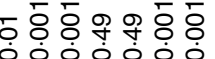

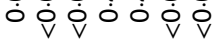

mo 눙 ம் ம் ळ ळ

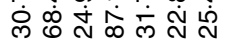


Table 2. Breakfast definitions

\section{Code Definition}

$\mathrm{Br} 1 \quad$ Anything consumed between 06.00 and 10.00 hours for weekdays or 06.00 and 11.00 hours for weekends ${ }^{(22)}$

Br2 Anything consumed between 05.00 and 10.00 hours with a combined total energy $\geq 418 \mathrm{~kJ}(\geq 100 \mathrm{kcal})^{(23)}$

Br3 Anything consumed between 05.00 and 10.00 hours for weekdays or 05.00 and 11.00 hours for weekends, excluding caffeinated drinks $\mathrm{Br} 4$ Anything consumed at home ${ }^{(24)}$

Br5 Consumed at home and included at least one solid food

Br6 Anything consumed at home, excluding caffeinated drinks

Br7 Anything consumed before starting daily activities

Br8 Consumed before starting daily activities and included at least one solid food

Br9 Consisting of something more than a glass of milk or fruit juice ${ }^{(25)}$

Br10 Consisting of something more than ready-to-eat cereals ${ }^{(26)}$

Br11 Consumed before starting daily activities, no later than 10.00 hours and of an energy level between 20 and $35 \%$ of total daily energy needs $^{(2)}$

Breakfast was defined by eleven different ways based on the two 24-h recalls. These definitions refer to the first eating episode of the day, taking into consideration the time of day, location, daily activities, types of food or beverages consumed or amount of energy provided. These breakfast definitions are described in Table 2.

Subjects who did not consume breakfast on both days were categorised as breakfast skippers. Subjects who consumed breakfast on one of the $2 \mathrm{~d}$ were categorised as occasional breakfast eaters, whereas those who consumed breakfast on both days were classified as breakfast eaters ${ }^{(23)}$. Breakfast consumption was also defined based on what a person perceives as a breakfast meal using a single question about breakfast consumption: "how often do you have breakfast?". Available answers were: "never/rarely", "1-3 times/month", "1-2 times/week", "3-6 times/week" and "7 times/week". Finally, the first eating episode was analysed in terms of food groups.

\section{Other variables}

Information on demographic parameters (age, sex) and anthropometric and weight-related characteristics (current and maximum weight, height, initial weight loss) were obtained through structured questionnaires. Physical activity was assessed by the short version of the International Physical Activity Questionnaire ${ }^{(27)}$, translated and validated in the Greek population ${ }^{(28)}$. Participants reported all domains of physical activity during the last $7 \mathrm{~d}$.

\section{Statistical analyses}

Characteristics of those who maintain the weight loss were compared with those who regained it. For non-normally distributed quantitative variables, the Mann-Whitney test was performed. For categorical data, Pearsons' $\chi^{2}$ test was used to check for differences between groups. The association between breakfast consumption and maintenance status was also assessed using binary logistic regression models that calculated OR. Model 1 was unadjusted; model 2 was adjusted for age; model 3 was further adjusted for total energy intake and adherence to the Mediterranean diet; model 4 was adjusted for age, total energy intake and intake of whole grains, fruits, sweets at breakfast meal; model 5 was adjusted for age, total energy intake and daily intake of whole grains, fruits and sweets; and model 6 was adjusted for age, total energy intake, adherence to Mediterranean diet and physical activity. Goodness of fit of the models was tested by the Hosmer and Lemeshow test. Data analysis was carried out using SPSS Statistics 22.0; a $P$-value of 0.05 was considered statistically significant.

\section{Results}

Daily energy intake did not differ between maintainers and regainers (7406 (SD 2724) v. $7778(\mathrm{SD} 2862) \mathrm{kJ}(1770$ (SD 651) $v$. 1859 (sD 684) kcal), respectively, $P=0 \cdot 142$ ), nor did diet quality, assessed by the MedDietScore (28.3 (SD 6.0) v. 28.2 (SD 6.0), respectively, $P=0.633)$. These findings were also true when men and women were analysed separately. Physical activity levels differed between male maintainers and regainers (2504 (1253-4893) v. 1283 (511-2714) total MET-min/week, respectively, $P=0 \cdot 001)$, whereas no such difference was observed in women (1555 (792-3073) v. 1400 (782-2946) total MET-min/ week, respectively, $P=0 \cdot 001)$. No differences were observed in sitting hours between maintainers and regainers in both sexes.

With regard to definitions of breakfast meal, the percentage of those consuming breakfast varied from 1.1 to $75.9 \%$ in maintainers and from 0 to $61.2 \%$ in regainers depending on the definition used. When breakfast was defined based on time of consumption (Br1), $63.8 \%$ of maintainers and $61.2 \%$ of regainers were classified as regular breakfast eaters. Using a definition based on time of consumption combined with energy content $(\mathrm{Br} 2)$, the percentages of regular breakfast eaters were 44.2 and $41.8 \%$ in maintainers and regainers, respectively, whereas when the types of food consumed were taken into account $(\mathrm{Br} 9)$ the percentages of regular breakfast eaters were even lower, as merely $21.1 \%$ of maintainers and $14.3 \%$ of regainers appeared to consume breakfast regularly. None of these between-group comparisons were statistically significant (Fig. 1).

Statistically significant differences between maintainers and regainers were observed only in two definitions of breakfast meal (Figs 1 and 2). Specifically, $74 \%$ of maintainers $v$. $60 \%$ of regainers consumed breakfast regularly when breakfast was defined by definition $\operatorname{Br} 4(P=0 \cdot 013)$. When breakfast was defined as $\mathrm{Br} 7$, $76 \%$ of maintainers $v .57 \%$ of regainers were regular breakfast eaters $(P=0.002)$. When men and women were analysed separately, female maintainers and regainers differed significantly only in $\mathrm{Br} 7$ definition (regular consumption frequencies of maintainers and regainers $77.2 v \cdot 57 \cdot 8 \%$, respectively, $P=0.013$ ). On the other hand, statistically significant differences in four breakfast definitions (i.e. $\mathrm{Br} 3, \mathrm{Br} 4, \mathrm{Br} 6$ definitions and self-reported breakfast consumption) were observed in male maintainers and regainers, with maintainers having higher frequencies of regular breakfast consumption. Specifically, frequencies of regular breakfast consumption for male maintainers and regainers were, respectively, $49.5 v .29 .4 \%$ for $\operatorname{Br} 3(P=0.046), 71 v .38 .2 \%$ for $\mathrm{Br} 4$ $(P=0.002), 58.9 v .32 \cdot 3 \%$ for $\operatorname{Br} 6(P=0.026)$ and $56.2 v .36 \cdot 8 \%$ for self-reported daily breakfast consumption $(P=0 \cdot 008)$. 


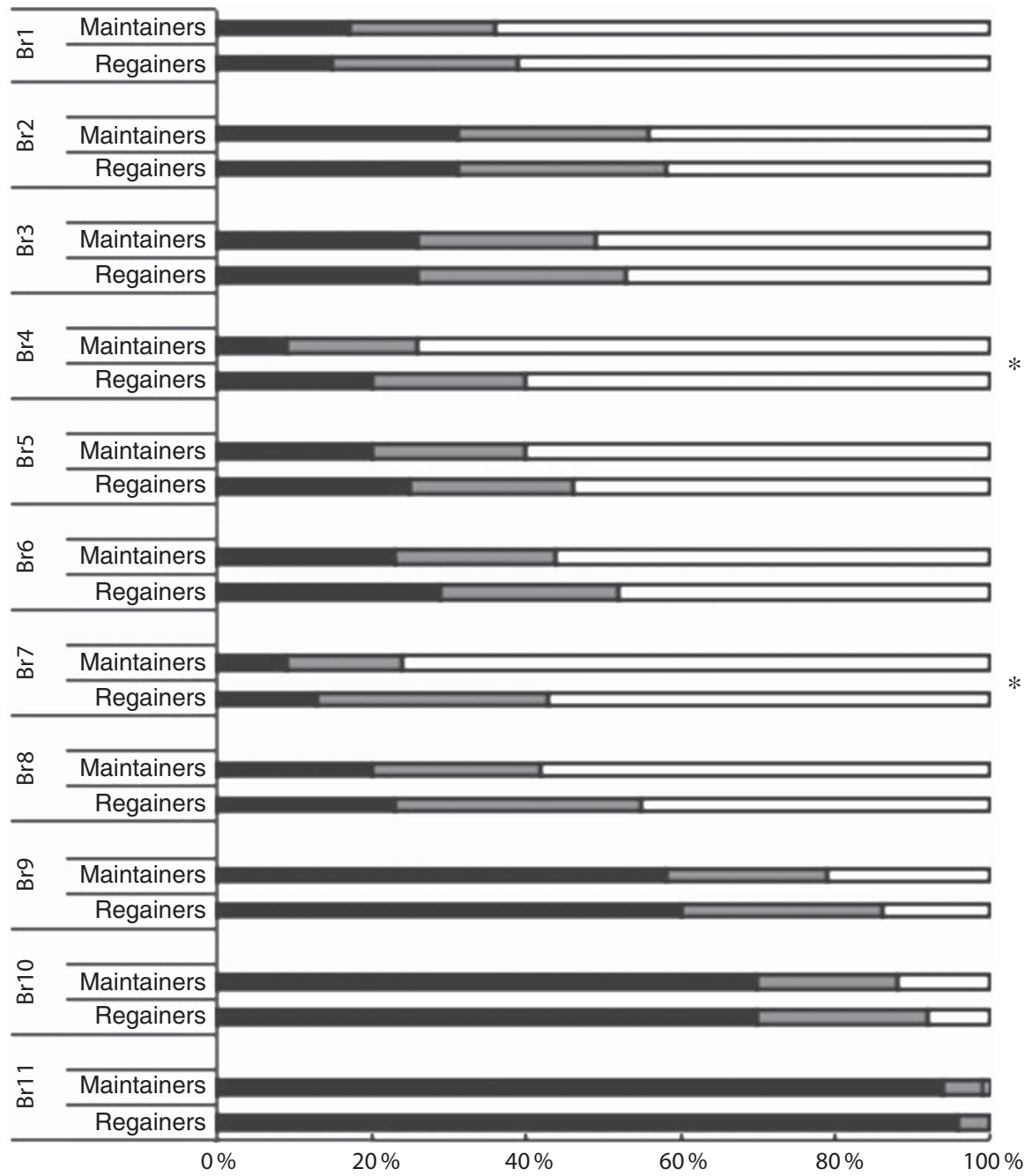

Fig. 1. Breakfast frequency according to maintenance status based on recall data (percentages). Mean values were significantly different at ${ }^{*} P<0.05$ (Pearson's $\chi^{2}$ test). $\square$, Breakfast skippers; $\square$, occasional breakfast eaters; $\square$, breakfast eaters.

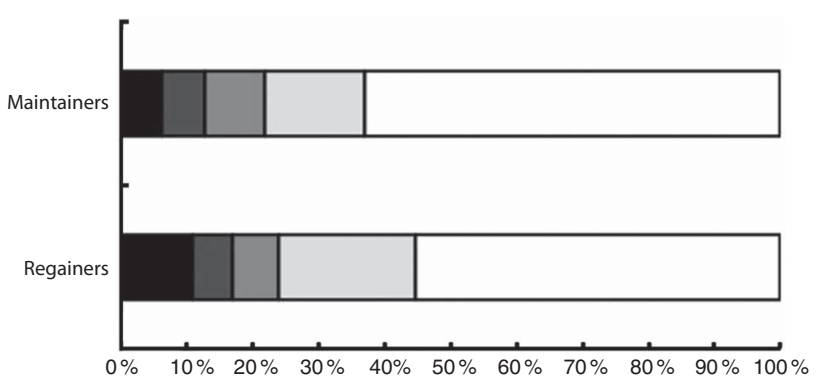

Fig. 2. Self-reported breakfast frequency according to maintenance status (percentages). No statistically significant differences were found $(P=0.310$, Pearson's $\chi^{2}$ test). $\square$, Rarely/never; $\square, 1-3$ times/month; $\square, 1-2$ times/week; $\square$, 3-6 times/week; $\square$, daily.

A logistic regression analysis was performed separately in men and women because of the interaction observed between sex and breakfast consumption. Results from this analysis showed that breakfast defined by Br7 definition was not associated with weight-loss maintenance in women. With regard to men, breakfast defined by $\operatorname{Br} 4$ or Br6 definition was associated with weight-loss maintenance. Specifically, the OR of being a maintainer for men consuming breakfast regularly compared with those skipping breakfast were 4.59 (95\% CI $1.72,12.30$, $P=0.03$ ) and 3.08 (95\% CI 1.24, 6.68, $P=0.03$ ) according to the above definitions, respectively. Self-reported breakfast consumption was also associated with weight-loss maintenance. The OR of being a maintainer among men consuming breakfast 1-2 times a week compared with those consuming breakfast rarely or never were 9.50 (95\% CI $1.50,60 \cdot 11, P=0 \cdot 01$ ), respectively. The association between the aforementioned breakfast definitions and weight-loss maintenance remained statistically significant after adjustment for age, total energy intake and adherence to Mediterranean diet; or for age, total energy intake and whole grain, fruit and sweet intake; or for age, total energy intake, adherence to Mediterranean diet and physical activity (online Supplementary Tables S3 and S4). The goodness of fit was checked by the Hosmer and Lemeshow test, and it was found that all models have a good fit.

Finally, breakfast defined as the first eating episode at home was further analysed in food groups. There was no statistically 
significant difference between maintainers and regainers in breakfast quality. No statistically significant difference was found between female maintainers and regainers in food groups consumed at breakfast meal. In men, however, those who regained weight had a higher intake of full-fat cheese at breakfast meal per day compared with those who maintained weight loss $(0.5$ $(0-1.25) v .0 \cdot 0(0 \cdot 0-0.5)$ daily portions of full-fat cheese, respectively, $P=0.007$ ) (one portion of full-fat cheese equals $30 \mathrm{~g}$ ).

\section{Discussion}

The present study is the first one that examined various breakfast definitions in relation to weight-loss maintenance in participants who had maintained or regained weight loss. We observed that breakfast consumption was positively associated with weight-loss maintenance only in men. Breakfast definitions able to identify weight-loss maintenance were the first eating episode consumed at home or consumed at home excluding caffeinated drinks, as well as the self-reported breakfast definition.

It is of interest that breakfast consumption was positively associated with weight-loss maintenance only in men. This finding may be because of the fact that breakfast consumption is more common in women ${ }^{(6)}$. Thus, perhaps women consume breakfast independently of their maintenance status. Indeed, the percentage of male maintainers consuming breakfast regularly is twice the percentage of regainers, and this percentage is almost equivalent to the percentage of women.

Breakfast consumption was positively associated with weight-loss maintenance when breakfast was defined as the first eating episode at home or consumed at home excluding caffeinated drinks even after adjusting for potential confounders such as age, overall diet quality and total daily energy intake. A theoretical model supporting a role for breakfast in the development of obesity has been proposed ${ }^{(2)}$. According to this model, breakfast consumption may lead to reduced total energy intake and improved diet quality, and therefore a reduced obesity risk. This explanation is not in agreement with our data, as breakfast consumption was found to be associated with weight-loss maintenance independently of total daily energy intake and diet quality. We also observed that breakfast consumption was related to weight-loss maintenance even after adjusting for physical activity, according to the aforementioned breakfast definitions. These findings imply that breakfast consumption itself maybe protects against regaining.

Lately, regular consumption of breakfast has been associated with health outcomes, such as obesity ${ }^{(6)}$ or reduced incidence of diabetes ${ }^{(29)}$ or $\mathrm{CHD}^{(30)}$, independently of $\mathrm{BMI}$, energy intake, physical activity and indicators of diet quality. It has been postulated that breakfast consumption may confer its health effects through ceasing prolonged fasting and thus by decreasing orexigenic stimuli (i.e. increased ghrelin levels in the fasting state) and by increasing anorexigenic stimuli (i.e. decreased insulin levels in the fasting state $)^{(6,29)}$. In accordance with the previous hypothesis, our data denote that maintainers, having breakfast at home, stop prolonged fasting sooner compared with regainers. Therefore, it is possible that breaking prolonged fasting through breakfast consumed at home may result in an improved appetite-related hormone status throughout the day and thus may lead to weight-loss maintenance. Further research is needed to elucidate potential mechanisms and pathways.

Breakfast quality may also affect long-term weight management, as a positive effect of whole-grain cereals and a negative effect of full-fat cheese on weight maintenance status were revealed in the present analysis. Data from the US National Weight Control Registry (NWCR) indicate that participants who manage to maintain weight loss usually consume cereals and fruits for breakfast ${ }^{(9)}$. Moreover, a recent clinical trial showed that participants consuming a high-carbohydrate breakfast showed better maintenance rates of weight loss ${ }^{(12)}$. Therefore, eating whole-grain cereals and avoiding full-fat cheese at breakfast meal possibly leads to maintaining weight loss.

Finally, based on what participants perceive as a breakfast meal, breakfast consumed 1-2 times/week or daily was positively associated with maintenance of weight loss. Daily consumption is a common characteristic among maintainers according to NWCR members, as $78 \%$ of registry members have reported this behaviour ${ }^{(9)}$. The present study is consistent with this finding: $56.2 \%$ of male maintainers compared with $36.8 \%$ of male regainers reported daily breakfast consumption. It should also be noted that statistical significance was lost after adjustment for total energy intake and adherence to Mediterranean diet. This could indicate that daily breakfast protects against regaining weight via reduced total energy intake. Indeed, breakfast consumption has been linked to total energy intake reduction ${ }^{(31,32)}$.

Strengths of the present study include the study sample that consisted of both maintainers and regainers. Furthermore, two 24-h dietary recalls were used in order to assess dietary intake, and breakfast consumption was assessed by twelve different definitions. It has, also, few limitations. The observational nature of the study indicates associations, but no causal inferences can be extracted from the present findings. All participant characteristics were self-reported; however, research indicates high concordance between objective and web-based selfreported measures of height and weight for adults ${ }^{(33)}$. Finally, participants were not equally distributed in the two groups, with the maintainer group being three times bigger compared with the regainer group. However, we do not have an indication that this unequal distribution could have affected our results.

In summary, the present study indicates that when breakfast is consumed at home it may influence weight-loss maintenance. Further research should be conducted to clarify whether this finding applies only in males, as indicated by our results, as well as the potential mechanisms and pathways by which breakfast consumption confers its health outcomes. Public health strategies for the promotion of breakfast as the first at-home meal of the day may support primary and secondary obesity prevention. This was not a prospective study, and thus we cannot conclude that breakfast eating was a cause of success in weight-loss maintainers. Future studies, prospectively linking breakfast eating with success in weight maintenance, would help clarify this relationship.

\section{Acknowledgements}

The MedWeight study was financially supported by the CocaCola Foundation (KA 221). D. B., E. K. and C. A. A have received 
stipends from this grant. E. K. has received financial support from the Greek State Scholarships Foundation. Coca-Cola Foundation and the Greek State Scholarships Foundation had no role in the design, analysis or writing of this article.

The authors' contributions are as follows: M. Y. and E. K. designed the research; D. B. and C. A. A. contributed to the analysis and interpretation of the data; and D. B. wrote the paper. All authors conducted the research, and read and approved the final manuscript.

The authors declare that there are no conflicts of interest.

\section{Supplementary material}

For supplementary material/s referred to in this article, please visit http://dx.doi.org/doi:10.1017/S0007114516001550

\section{References}

1. Dialektakou KD \& Vranas PB (2008) Breakfast skipping and body mass index among adolescents in Greece: whether an association exists depends on how breakfast skipping is defined. J Am Diet Assoc 108, 1517-1525.

2. Timlin MT \& Pereira MA (2007) Breakfast frequency and quality in the etiology of adult obesity and chronic diseases. Nutr Rev 65, 268-281.

3. Casazza K, Pate R \& Allison DB (2013) Myths, presumptions, and facts about obesity. N Engl J Med 368, 2236-2237.

4. Brown AW, Bohan Brown MM \& Allison DB (2013) Belief beyond the evidence: using the proposed effect of breakfast on obesity to show 2 practices that distort scientific evidence. Am J Clin Nutr 98, 1298-1308.

5. So HK, Nelson EA, Li AM, et al. (2011) Breakfast frequency inversely associated with BMI and body fatness in Hong Kong Chinese children aged 9-18 years. Br J Nutr 106, 742-751.

6. Odegaard AO, Jacobs DR Jr., Steffen LM, et al. (2013) Breakfast frequency and development of metabolic risk. Diabetes Care 36, 3100-3106.

7. van der Heijden AA, Hu FB, Rimm EB, et al. (2007) A prospective study of breakfast consumption and weight gain among U.S. men. Obesity (Silver Spring) 15, 2463-2469.

8. Purslow LR, Sandhu MS, Forouhi N, et al. (2008) Energy intake at breakfast and weight change: prospective study of 6,764 middle-aged men and women. Am J Epidemiol 167, $188-192$.

9. Wyatt HR, Grunwald GK, Mosca CL, et al. (2002) Long-term weight loss and breakfast in subjects in the National Weight Control Registry. Obes Res 10, 78-82.

10. Dhurandhar EJ, Dawson J, Alcorn A, et al. (2014) The effectiveness of breakfast recommendations on weight loss: a randomized controlled trial. Am J Clin Nutr 100, 507-513.

11. Jakubowicz D, Barnea M, Wainstein J, et al. (2013) High caloric intake at breakfast vs. dinner differentially influences weight loss of overweight and obese women. Obesity (Silver Spring) 21, 2504-2512.

12. Jakubowicz D, Froy O, Wainstein J, et al. (2012) Meal timing and composition influence ghrelin levels, appetite scores and weight loss maintenance in overweight and obese adults. Steroids 77, 323-331.

13. Affenito SG (2007) Breakfast: a missed opportunity. J Am Diet Assoc 107, 565-569.

14. O'Neil CE, Byrd-Bredbenner C, Hayes D, et al. (2014) The role of breakfast in health: definition and criteria for a quality breakfast. J Acad Nutr Diet 114, Suppl., S8-S26.
15. Karfopoulou E, Anastasiou CA, Hill JO, et al. (2014) The MedWeight study: design and preliminary results. Med J Nutr Metab 7, 201-210.

16. Thompson FE \& Byers T (1994) Dietary assessment resource manual. J Nutr 124, Suppl., 2245S-2317S.

17. Tran KM, Johnson RK, Soultanakis RP, et al. (2000) In-person vs telephone-administered multiple-pass 24-hour recalls in women: validation with doubly labeled water. J Am Diet Assoc 100, $777-783$.

18. Fardet A \& Boirie Y (2014) Associations between food and beverage groups and major diet-related chronic diseases: an exhaustive review of pooled/meta-analyses and systematic reviews. Nutr Rev 72, 741-762.

19. Fogelholm M, Anderssen S, Gunnarsdottir I, et al. (2012) Dietary macronutrients and food consumption as determinants of long-term weight change in adult populations: a systematic literature review. Food Nutr Res 56, 10.3402/fnr.v56i0.19103.

20. Willett WC, Sacks F, Trichopoulou A, et al. (1995) Mediterranean diet pyramid: a cultural model for healthy eating. $A m \mathrm{~J}$ Clin Nutr 61, Suppl., 1402S-1406S.

21. Panagiotakos DB, Pitsavos C \& Stefanadis C (2006) Dietary patterns: a Mediterranean diet score and its relation to clinical and biological markers of cardiovascular disease risk. Nutr Metab Cardiovasc Dis 16, 559-568.

22. Aranceta J, Serra-Majem L, Ribas L, et al. (2001) Breakfast consumption in Spanish children and young people. Public Health Nutr 4, 1439-1444

23. Alexander KE, Ventura EE, Spruijt-Metz D, et al. (2009) Association of breakfast skipping with visceral fat and insulin indices in overweight Latino youth. Obesity (Silver Spring) 17, 1528-1533.

24. Keski-Rahkonen A, Kaprio J, Rissanen A, et al. (2003) Breakfast skipping and health-compromising behaviors in adolescents and adults. Eur J Clin Nutr 57, 842-853.

25. Vereecken C, Dupuy M, Rasmussen M, et al. (2009) Breakfast consumption and its socio-demographic and lifestyle correlates in schoolchildren in 41 countries participating in the HBSC study. Int J Public Health 54, Suppl. 2, 180-190.

26. Sjoberg A, Hallberg L, Hoglund D, et al. (2003) Meal pattern, food choice, nutrient intake and lifestyle factors in The Goteborg Adolescence Study. Eur J Clin Nutr 57, 1569-1578.

27. Craig CL, Marshall AL, Sjostrom M, et al. (2003) International physical activity questionnaire: 12-country reliability and validity. Med Sci Sports Exerc 35, 1381-1395.

28. Papathanasiou G, Georgoudis G, Papandreou M, et al. (2009) Reliability measures of the short International Physical Activity Questionnaire (IPAQ) in Greek young adults. Hellenic J Cardiol 50, 283-294.

29. Mekary RA, Giovannucci E, Cahill L, et al. (2013) Eating patterns and type 2 diabetes risk in older women: breakfast consumption and eating frequency. Am J Clin Nutr 98, 436-443.

30. Cahill LE, Chiuve SE, Mekary RA, et al. (2013) Prospective study of breakfast eating and incident coronary heart disease in a cohort of male US health professionals. Circulation 128, 337-343.

31. Farshchi HR, Taylor MA \& Macdonald IA (2005) Deleterious effects of omitting breakfast on insulin sensitivity and fasting lipid profiles in healthy lean women. Am J Clin Nutr 81, 388-396.

32. Azadbakht L, Haghighatdoost F, Feizi A, et al. (2013) Breakfast eating pattern and its association with dietary quality indices and anthropometric measurements in young women in Isfahan. Nutrition 29, 420-425.

33. Lassale C, Peneau S, Touvier M, et al. (2013) Validity of web-based self-reported weight and height: results of the Nutrinet-Sante study. J Med Internet Res 15, e152. 